\title{
Hot gas in Mach cones around Virgo cluster spiral galaxies ${ }^{\star}$
}

\author{
M. Weżgowiec ${ }^{1}$, B. Vollmer ${ }^{2}$, M. Ehle ${ }^{3}$, R.-J. Dettmar ${ }^{1}$, D. J. Bomans ${ }^{1}$, K. T. Chyży ${ }^{4}$, \\ M. Urbanik ${ }^{4}$, and M. Soida ${ }^{4}$
}

\begin{abstract}
1 Astronomisches Institut der Ruhr-Universität Bochum, Universitätsstrasse 150, 44780 Bochum, Germany e-mail: mawez@astro.rub.de

2 CDS, Observatoire astronomique de Strasbourg, UMR7550, 11 rue de l'université, 67000 Strasbourg, France

3 ESAC, XMM-Newton Science Operations Centre, PO Box 78, 28691 Villanueva de la Cañada, Madrid, Spain

${ }^{4}$ Obserwatorium Astronomiczne Uniwersytetu Jagiellońskiego, ul. Orla 171, 30-244 Kraków, Poland
\end{abstract}

Received 17 December 2010 / Accepted 8 April 2011

ABSTRACT

\begin{abstract}
Context. The detailed comparison between observations and simulations of ram-pressure stripped spiral galaxies in the Virgo cluster has led to a 3D view of the galaxy orbits within the hot intracluster medium. The 3D velocities and Mach numbers derived from simulations can be used to derive simple Mach cone geometries for Virgo spiral galaxies.

Aims. We search for indications of hot gas within Mach cones in X-ray observations of selected Virgo cluster spiral galaxies (NGC 4569, NGC 4388, and NGC 4501).

Methods. Low-resolution maps of diffuse extended emission and X-ray spectra from XMM-Newton observations are presented. Gas densities and temperatures were derived from the X-ray spectra.

Results. We find extraplanar diffuse X-ray emission in all galaxies. Based on the 3D velocity vectors from dynamical modeling, a simple Mach cone is fitted to the triangular shape of NGC 4569's diffuse X-ray emission. Assuming that all extraplanar diffuse X-ray emission has to be located inside the Mach cone, we also fit Mach cones to NGC 4388's and NGC 4501's extraplanar X-ray emission. For NGC 4569 it is hard to reconcile the derived Mach cone opening angle with a Mach number based on the sound speed alone. Instead, a Mach number involving the Alfvénic speed seems to be more appropriate, yielding a magnetic field strength of $\sim 1-3 \mu \mathrm{G}$ for an intracluster medium density of $n \sim 10^{-4} \mathrm{~cm}^{-3}$. Whereas the temperature of the hot component of NGC 4569's X-ray halo (0.5 keV) is at the high end, but typical of a galactic outflow, the temperature of the hot gas tails of NGC 4388 and NGC 4501 are significantly hotter $(0.7-0.9 \mathrm{keV})$.

Conclusions. In NGC 4569 we find direct evidence of a Mach cone that is filled with hot gas from a galactic superwind. We suggest that the high gas temperatures in the X-ray tails of NGC 4388 and NGC 4501 come from the mixing of the stripped ISM into the hot intracluster medium of the Virgo cluster.
\end{abstract}

Key words. galaxies: clusters: general - galaxies: clusters: individual: Virgo - galaxies: individual: NGC 4388 galaxies: individual: NGC 4501 - galaxies: individual: NGC 4569 - galaxies: clusters: intracluster medium

\section{Introduction}

There is ample evidence that the cluster environment modifies the gas distribution of spiral galaxies. Especially in the Virgo cluster, where the gas distribution of spiral galaxies can be observed in detail by current interferometric observations, radially truncated gas disks are frequently observed (Cayatte et al. 1990; Chung et al. 2009). The Virgo cluster has the advantage of being spiral-rich and dynamically young; i.e., there are strong ongoing environmental interactions. Chung et al. (2007) found seven spiral galaxies with long $\mathrm{HI}$ tails in intermediate-to-low galaxy-density regions $(0.6-1 \mathrm{Mpc}$ in projection from M 87). The tails are all pointing roughly away from M 87. Assuming radial galaxy orbits, this suggests that these tails come from rampressure stripping, which therefore already begins to affect spiral galaxies around the cluster Virial radius. The further evolution of a galaxy depends critically on its orbit (see, e.g., Vollmer et al. 2001); i.e., a highly eccentric orbit will lead the galaxy at a high velocity into the cluster core, where the intracluster medium is densest and ram-pressure will be very strong. Recently,

* Based on observations obtained with XMM-Newton, an ESA science mission with instruments and contributions directly funded by ESA Member States and NASA.
Vollmer (2009) established a model-based ram-pressure stripping time sequence of Virgo spiral galaxies. Snapshots of 3D models including ram-pressure were compared to the observed Hi gas distributions and velocity fields. The 3D velocity vector from the dynamical model and the timestep of the best-fit model has to be consistent with the galaxy's projected position and radial velocity within the Virgo cluster. Moreover, Vollmer (2009) concluded that the linear orbital segments derived from the dynamical models, together with the intracluster medium density distribution derived from X-ray observations, are consistent with the dynamical simulations of 6 Virgo cluster spirals. The deduced closest approach of the galaxies to the cluster center (M 87) is between 200 and $600 \mathrm{kpc}$.

Another consequence of ongoing ram-pressure can be found in the distribution of polarized radio continuum emission, which is sensitive to gas compression and shear motions. Asymmetric ridges of polarized radio continuum emission are frequently observed in Virgo spiral galaxies with disturbed or truncated Hi disks (Weżgowiec et al. 2007; Vollmer et al. 2007). MHD modeling based on the velocity fields of the dynamical model can corroborate a ram-pressure stripping scenario and/or distinguish between different interaction scenarios (NGC 4654, Soida et al. 2006; NGC 4522, Vollmer et al. 2006; NGC 4501, Vollmer et al. 2008). 
Table 1. Basic astronomical properties of studied galaxies.

\begin{tabular}{|c|c|c|c|c|c|c|c|}
\hline \multirow[t]{2}{*}{ Name } & \multirow{2}{*}{$\begin{array}{l}\text { Morph. } \\
\text { type }^{a}\end{array}$} & \multicolumn{2}{|c|}{ Optical position ${ }^{a}$} & \multirow{2}{*}{$\begin{array}{c}\text { Incl. }^{a} \\
\left.{ }^{\circ}\right] \\
\end{array}$} & \multirow{2}{*}{$\begin{array}{c}\text { Pos. ang. }{ }^{a} \\
{\left[{ }^{\circ}\right]}\end{array}$} & \multirow{2}{*}{$\begin{array}{l}\text { Proj. dist. } \\
\text { to Vir A }\left[^{\circ}\right]\end{array}$} & \multirow{2}{*}{$\begin{array}{c}N_{\mathrm{H}}^{b} \\
{\left[10^{20} \mathrm{~cm}^{-2}\right]}\end{array}$} \\
\hline & & $\alpha_{2000}$ & $\delta_{2000}$ & & & & \\
\hline NGC 4388 & $\mathrm{Sb}$ & $12^{\mathrm{h}} 25^{\mathrm{m}} 46^{\mathrm{s}} .8$ & $+12^{\circ} 39^{\prime} 44^{\prime \prime}$ & 82 & 91 & 1.3 & 2.58 \\
\hline NGC 4501 & $\mathrm{Sb}$ & $12^{\mathrm{h}} 31^{\mathrm{m}} 59^{\mathrm{s}} 3$ & $+14^{\circ} 25^{\prime} 14^{\prime \prime}$ & 60 & 138 & 2.0 & 2.62 \\
\hline NGC 4569 & $\mathrm{SABa}$ & $12^{\mathrm{h}} 36^{\mathrm{m}} 50^{\mathrm{s}} .1$ & $+13^{\circ} 09^{\prime} 46^{\prime \prime}$ & 66 & 23 & 1.7 & 2.82 \\
\hline
\end{tabular}

Notes. ${ }^{(a)}$ HYPERLEDA database - http://leda . univ-lyon1. fr - see Paturel et al. (2003); ${ }^{(b)}$ weighted average value following LAB Survey of Galactic $\mathrm{H}_{\mathrm{I}}$, see Kalberla et al. (2005).

Table 2. Parameters of X-ray observations of studied galaxies.

\begin{tabular}{lccccccc}
\hline \hline Name & Obs. ID & Obs. date & Exp. time $^{a}$ & pn filter pn obs. $^{b}$ & MOS filter & MOS obs. $^{b}$ \\
\hline NGC 4388 & 0110930701 & $2002-12-12$ & $11.9(6.9)$ & Thin & EF & Medium & FF \\
NGC 4501 & 0112550801 & $2001-12-04$ & $14(2.9)$ & Medium & EF & Thin & FF \\
NGC 4569 & 0200650101 & $2004-12-13 / 14$ & $66(49)$ & Thin & EF & Medium & FF \\
\hline
\end{tabular}

Notes. ${ }^{(a)}$ Total time in ks with clean time for pn camera in brackets. ${ }^{(b)}$ Observing mode: FF - Full Frame, EF - Extended Full Frame.

Despite these clear signs of ram-pressure stripping on the atomic gas content, only a handful of gas tails associated to cluster spiral galaxies have been detected in X-rays: in ACO 2125 (C153 at $z=0.253$, Wang et al. 2004), in ACO 1367 (UGC 6697 at $z=0.022$, Sun \& Vikhlinin 2005), in the Coma cluster (NGC 4848, Finoguenov et al. 2004), and in ACO 3627 (ESO 137-001, Sun et al. 2006). During a ram-pressure stripping event, gas is expected to heat up to X-ray temperatures by bow shocks, heat conduction, and the mixing of the stripped ISM into the hot intracluster medium (e.g., Stevens et al. 1999; Schulz \& Struck 2001; Roediger et al. 2006; Roediger \& Brüggen 2008; Tonnesen \& Bryan 2009), so it is rather surprising and not clear why the X-ray detection rate is so low. In particular, there is no detection of the leading bow shock of a cluster spiral galaxy in the literature to our knowledge. Rasmussen et al. (2006) detected a shock-like feature in NGC 2276 which is part of the NGC 2300 group of galaxies. Since galaxies move with velocities between 1000 and $2000 \mathrm{~km} \mathrm{~s}^{-1}$ and the sound speed in the intracluster medium of the Virgo cluster is $\sim 500-700 \mathrm{~km} \mathrm{~s}^{-1}$, a bow shock with an associated Mach cone is expected.

In this article we have used XMM-Newton data to search for $\mathrm{X}$-ray signatures of $3 \mathrm{ram}$-pressure stripped spiral galaxies belonging to the ram-pressure stripping sequence from Vollmer (2009). We made deep XMM-Newton spectroscopic observations of NGC 4569 and used archival XMM-Newton data for NGC 4388 and NGC 4501. They are described in Sect. 2, and the results are presented in Sect. 3. We find extraplanar diffuse gas in all galaxies. Simple Mach cone geometries are fitted to the X-ray images, and the thermal pressure is compared to ramand cosmic ray pressure in Sect. 4. We give our conclusions in Sect. 5 .

\section{Observations}

We made deep XMM-Newton observations of NGC 4569 (proposal ID: 2006501, PI: M. Ehle). In addition, archival XMM-Newton observation of NGC 4388 and NGC 4501 were used. The main astronomical parameters of the studied galaxies are presented in Table 1 and the properties of their X-ray observations in Table 2. The data were processed using the SAS 9.0 package (Gabriel et al. 2004) with standard reduction procedures. Following the routine of tasks epchain and emchain calibrated event lists for the pn camera (Strüder et al. 2001) and for the two MOS cameras (Turner et al. 2001) were obtained for each galaxy. Next, the event lists were carefully filtered for bad CCD pixels and periods of intense radiation of high-energy background. The filtered event lists were used to produce images, background images, and exposure maps (without and with vignetting correction) and masked for an acceptable detector area using the images script ${ }^{1}$. All images and maps were produced in the band of $0.2-1 \mathrm{keV}$ in order to be sensitive to the softest $\mathrm{X}$-ray emission due to hot gas. Next, the final images were combined using the data from all cameras. The resulting images were adaptively smoothed with a Gaussian beam of $10^{\prime \prime}$ HPBW. To get a better signal-to-noise ratio and thus better sensitivity to extended structures, which allowed bringing up the large-scale structure, the images were again smoothed, this time with a Gaussian beam of $30^{\prime \prime}$ or $1^{\prime}$ with the AIPS package. The gaps between instrument detectors were not interpolated, but filled by combining scaled MOS and pn maps.

For the spectral analysis, the images were searched for pointlike sources using the routine task edetect_chain. Next, after excluding circular areas around the detected sources from the relevant region area, a spectrum was acquired. The sizes of circular areas were determined by visual inspection of an image of each point source in the pn event list. Blank-sky event lists (see Carter $\&$ Read 2007) were used for creating background spectrum corresponding to a proper region. For each spectrum response matrices and effective area files were produced. For the latter, the detector maps were also created for extended emission analysis. Finally, the spectra were then fitted using XSPEC 11 (Arnaud 1996). The thermal plasma MEKAL model is based on calculations by Mewe and Kaastra (Mewe et al. 1985; Kaastra 1992).

\section{Results}

In the following we present the $\mathrm{X}$-ray distribution in the band of $0.2-1 \mathrm{keV}$ and X-ray spectra of NGC 4569 , NGC 4388, and NGC 4501 from regions presented in Appendix A. The unabsorbed X-ray fluxes, X-ray luminosities, gas densities, and temperatures are presented in Table 3. The volumes of emitting

\footnotetext{
${ }^{1}$ http://xmm.esac.esa.int/external/xmm_science/ gallery/utils/images. shtml
} 
M. Weżgowiec et al.: Hot gas in Mach cones around Virgo cluster spiral galaxies

Table 3. X-ray fluxes, derived densities, and temperatures from the X-ray spectra.

\begin{tabular}{lccccccc}
\hline \hline Galaxy & Region & $\begin{array}{c}\text { Hot gas flux } \\
{\left[10^{-14} \mathrm{erg} \mathrm{cm}^{-2} \mathrm{~s}^{-1}\right]}\end{array}$ & $\begin{array}{c}L_{\mathrm{X}} \\
{\left[10^{39} \mathrm{erg} \mathrm{s}^{-1}\right]}\end{array}$ & $\begin{array}{c}\left(n_{\text {cold }} \eta^{0.5}\right)^{b} \\
{\left[10^{-3} \mathrm{~cm}^{-3}\right]}\end{array}$ & $\begin{array}{c}k T_{\text {cold }} \\
{[\mathrm{keV}]}\end{array}$ & $\begin{array}{c}\left(n_{\text {hot }} \eta^{0.5}\right)^{b} \\
{\left[10^{-3} \mathrm{~cm}^{-3}\right]}\end{array}$ & $\begin{array}{c}k T_{\text {hot }} \\
{[\mathrm{keV}]}\end{array}$ \\
\hline NGC & disk & $2.26_{-1.35}^{+1.48}$ & 0.780 & $1.6_{-0.8}^{+1.2}$ & $0.14_{-0.04}^{+0.10}$ & $2.0_{-0.6}^{+0.5}$ & $0.46 \pm 0.08$ \\
4569 & halo & $11.39_{-4.39}^{+5.28}$ & 3.936 & $0.7_{-0.2}^{+0.3}$ & $0.17_{-0.03}^{+0.02}$ & $0.7_{-0.2}^{+0.2}$ & $0.49 \pm 0.05$ \\
\hline NGC & nucleus & $16.84_{-8.92}^{+6.40}$ & 5.819 & $27.8_{-10.6}^{+13.7}$ & $0.14_{-0.03}^{+0.07}$ & $18.8_{-6.2}^{+5.2}$ & $0.66_{-0.04}^{+0.10}$ \\
4388 & disk & $10.44_{-10.44}^{+9.77}$ & 3.608 & $4.6_{-1.3}^{+3.3}$ & $0.21 \pm 0.03$ & $3.7_{-1.0}^{+1.0}$ & $0.79 \pm 0.11$ \\
& tail & $21.51_{-8.82}^{+5.92}$ & 7.433 & $0.7_{-0.3}^{+0.3}$ & $0.87_{-0.12}^{+0.13}$ & $0.9_{-0.9}^{+2.6}$ & $2.26_{-0.85}^{+2.16}$ \\
\hline NGC & disk & $16.44_{-6.03}^{+8.31}$ & 5.681 & - & - & $3.4_{-1.0}^{+1.0}$ & $0.38_{-0.07}^{+0.12}$ \\
4501 & tail & $14.31_{-8.87}^{+4.25}$ & 4.945 & - & - & $0.6_{-0.2}^{+0.2}$ & $0.67_{-0.09}^{+0.11}$ \\
\hline
\end{tabular}

Notes. ${ }^{(a)}$ Total unabsorbed flux from the thermal emission between 0.3 and $12 \mathrm{keV} .{ }^{(b)} \eta$ is the volume filling factor.

Table 4. Estimated volumes and gas masses.

\begin{tabular}{lcccc}
\hline \hline Galaxy & Region & $\begin{array}{c}\text { volume } \\
{\left[\mathrm{pc}^{3}\right]}\end{array}$ & $\begin{array}{c}\left(M_{\text {gas }}^{\text {cold }} \eta^{0.5}\right)^{a} \\
{\left[M_{\odot}\right]}\end{array}$ & $\begin{array}{c}\left(M_{\text {gas }}^{\text {hot }} \eta^{0.5}\right)^{a} \\
{\left[M_{\odot}\right]}\end{array}$ \\
\hline NGC & disk & $1.77 \mathrm{e}+11$ & $6.6_{-4.6}^{+5.6} \mathrm{e}+06$ & $7.8_{-4.6}^{+4.5} \mathrm{e}+06$ \\
4569 & halo & $6.14 \mathrm{e}+12$ & $9.8_{-5.7}^{+6.0} \mathrm{e}+07$ & $1.0_{-0.6}^{+0.6} \mathrm{e}+08$ \\
\hline NGC & nucleus & $1.10 \mathrm{e}+10$ & $6.9_{-4.3}^{+4.8} \mathrm{e}+06$ & $4.7_{-2.8}^{+2.7} \mathrm{e}+06$ \\
4388 & disk & $4.52 \mathrm{e}+11$ & $4.6_{-2.7}^{+2.7} \mathrm{e}+07$ & $3.7_{-2.1}^{+2.1} \mathrm{e}+07$ \\
& tail & $1.38 \mathrm{e}+13$ & $2.1_{-1.4}^{+1.4} \mathrm{e}+08$ & $2.8_{-3.1}^{+8.1} \mathrm{e}+08$ \\
\hline NGC & disk & $7.10 \mathrm{e}+11$ & - & $5.4_{-3.1}^{+3.2} \mathrm{e}+07$ \\
4501 & tail & $1.91 \mathrm{e}+13$ & - & $2.5_{-1.4}^{+1.4} \mathrm{e}+08$ \\
\hline
\end{tabular}

Notes. ${ }^{(a)} \eta$ is the volume filling factor.

regions were estimated in the following way (see Appendix A): (i) the galactic disk regions are approximated by cylinders with radii set by the extent of X-ray emission and a height of $1 \mathrm{kpc}$, (ii) the halo emission of NGC 4569 is assumed to be spherical with a radius of $11.4 \mathrm{kpc}$, (iii) the tail region of NGC 4388 is approximated by a cylinder with a length of $50.8 \mathrm{kpc}$ and a radius of $9.3 \mathrm{kpc}$, and (iv) the tail region of NGC 4501 is approximated by a cylinder with a length of $30 \mathrm{kpc}$ and a radius of $14 \mathrm{kpc}$. The model types and reduced $\chi^{2}$ are shown in Table 5. The volumes are uncertain by roughly a factor of 2 . The typical uncertainty of the density is $30-50 \%$ and of the masses $\sim 0.2$ dex. The volumes and gas masses of the different X-ray emitting components are presented in Table 4.

\subsection{NGC 4569}

The diffuse X-ray emission associated to NGC 4569 is extended over an area of approximately $20^{\prime} \times 20^{\prime}(100 \times 100 \mathrm{kpc}$; Fig. 1). The overall shape of the X-ray emission is that of a triangle with the tip pointing to the northeast. The galactic disk has a five times lower X-ray flux than the giant diffuse halo, which also encompasses a dwarf satellite galaxy IC 3583 . The large radio lobes observed at $20 \mathrm{~cm}$ (Chyży et al. 2006) are located entirely within the diffuse X-ray halo. There is enhanced X-ray emission associated to the southern half of the western radio lobe (at $\mathrm{RA}=12^{\mathrm{h}} 36^{\mathrm{m}} 30^{\mathrm{s}}$ Dec $\left.=13^{\circ} 09^{\prime}\right)$.

$\mathrm{X}$-ray spectra (Fig. 2) have been taken from the disk and the inner halo $\left(2.5^{\prime} \times 2.5^{\prime}\right.$ or $\left.12 \times 12 \mathrm{kpc}\right)$ excluding emission from the galactic disk. They are fitted by a two-temperature thermal plasma MEKAL model with a hot and a cold component. An additional powerlaw component is added to account for undetected point sources. Both the disk and halo show a cold $(\sim 0.15 \mathrm{keV})$ and a hot component $(\sim 0.5 \mathrm{keV})$ and densities of $n \sim 10^{-3} \eta^{-0.5} \mathrm{~cm}^{-3}$ (Table 3), where $\eta$ is the volume filling factor.
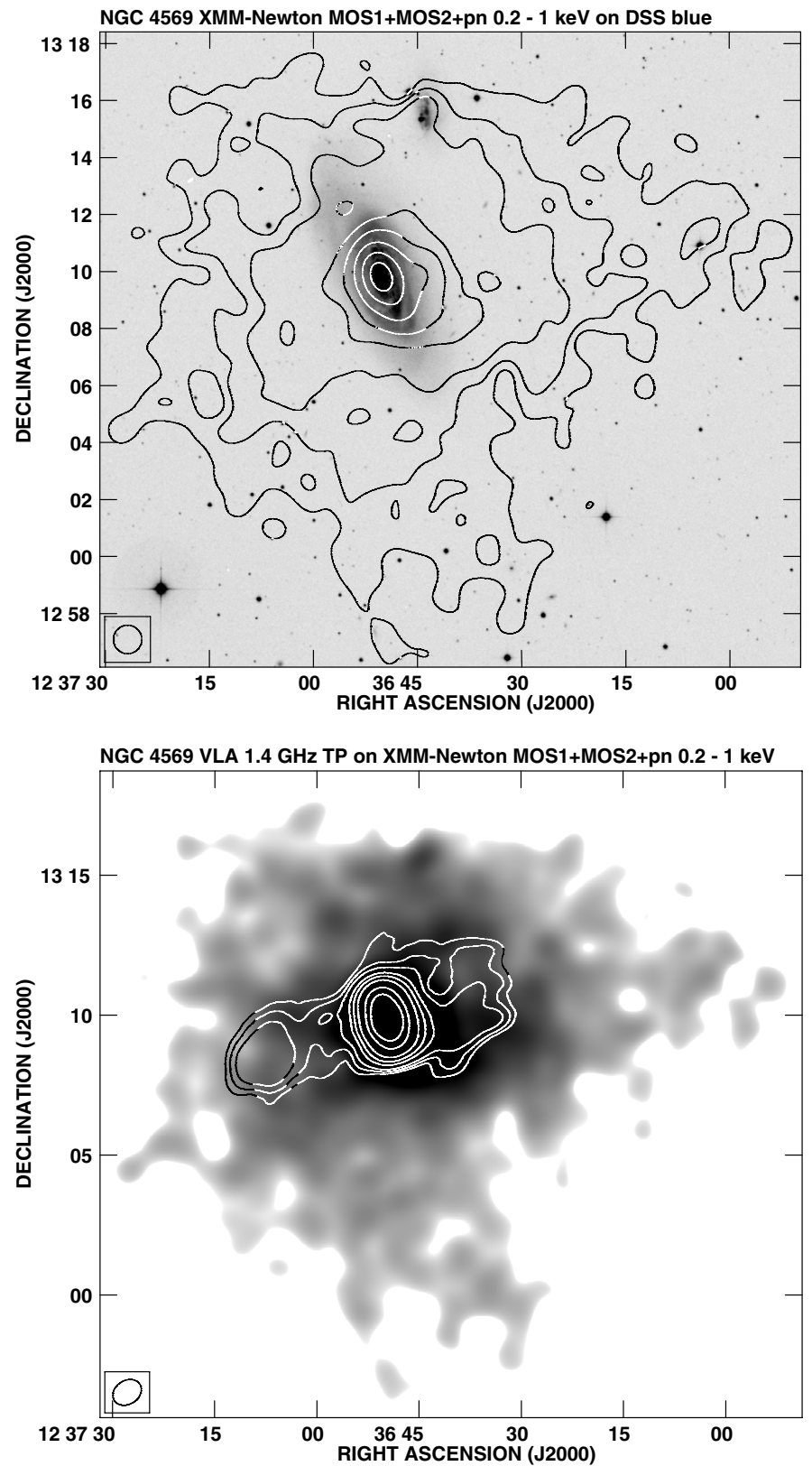

Fig. 1. X-ray emission $(0.2-1 \mathrm{keV})$ of NGC 4569 , together with an optical $B$ band (upper panel) and the radio total power map at $1.4 \mathrm{GHz}$ from Chyży et al. (2006; lower panel). The resolution of both maps is $1^{\prime}$. The beam size is shown in the bottom left corner of the figure. The first greyscale in the lower panel corresponds to 3 times the rms noise level. The radio contour levels are 3, 5, 8, 16, 25, 40, 80, $120 \times 0.24 \mathrm{mJy}$. 
Table 5. Model type and reduced $\chi_{\text {red }}^{2}$.

\begin{tabular}{lcc}
\hline \hline Galaxy & model type & $\chi_{\text {red }}^{2}$ \\
\hline NGC 4569 disk & wabs(mekal+mekal+powerlaw) & 0.96 \\
NGC 4569 halo & wabs(mekal+mekal+powerlaw) & 1.23 \\
NGC 4388 nucleus & wabs(mekal+mekal+powerlaw+wabs(powerlaw+Gauss)) & 1.06 \\
NGC 4388 disk+nucleus & wabs(mekal+mekal+powerlaw+wabs(powerlaw+Gauss)) & 1.07 \\
NGC 4388 tail & wabs(mekal+mekal+powerlaw) & 1.11 \\
NGC 4501 disk & wabs(mekal+powerlaw) & 1.39 \\
NGC 4501 tail & wabs(mekal+powerlaw) & 0.46 \\
\hline
\end{tabular}
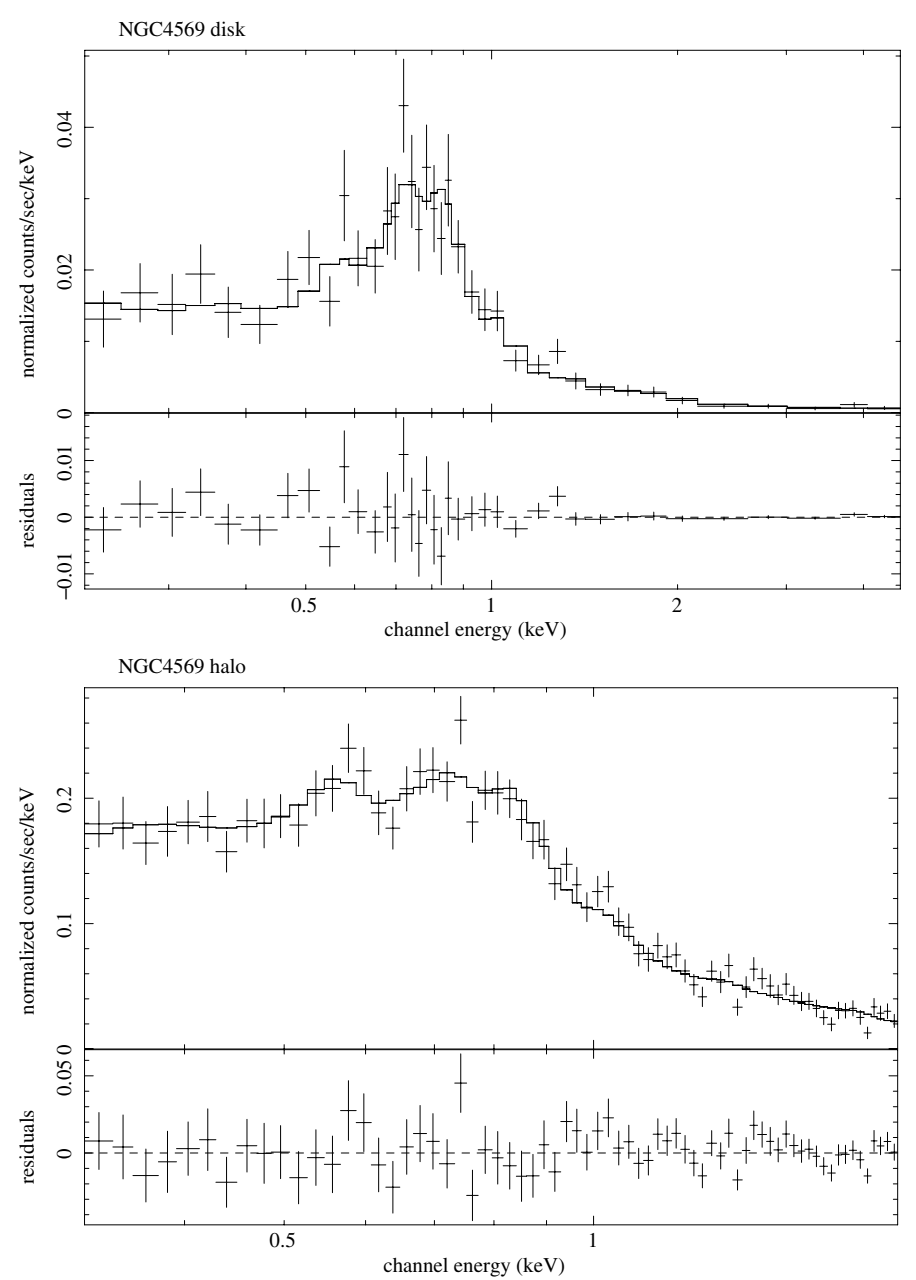

Fig. 2. X-ray spectrum of NGC 4569's disk (upper panel) and inner halo, excluding emission from the galactic disk (lower panel).

\subsection{NGC 4388}

The galactic disk of NGC 4388 is detected in X-rays. It is more extended to the north than to the south. In addition, an extended tail is detected towards the northeast of the galactic disk. This $\mathrm{X}$-ray tail is associated with the $\mathrm{H} \alpha$ plume reported by Yoshida et al. (2002) and the beginning of the huge Hi tail discovered by Oosterloo \& van Gorkom (2005, Fig. 3). At distances $>5^{\prime}$ from the galactic disk the X-ray emission starts to be dominated by the bright halo of M 86.

We performed a spectral analysis of the nucleus, the disk and nucleus, and the part of the tail close to the galactic disk. The spectra (Fig. 4) are fitted with two single temperature thermal plasma MEKAL models with an additional powerlaw component to account for undetected point sources. In the fit of the disk and the nucleus, a contribution from the AGN was included by adding an absorbed powerlaw component and the $6.4 \mathrm{keV}$ iron line to the model (see Table 5). The temperatures and densities are given in Table 3 . The nucleus has a gas density of $\sim 30 \eta^{-0.5} \mathrm{~cm}^{-3}$. The cold and hot components of the disk have temperatures of $\sim 0.2 \mathrm{keV}$ and $\sim 0.8 \mathrm{keV}$. The exceptionally high temperature of the hot component in the disk is probably due to the nuclear outflow (Veilleux et al. 1999; Yoshida et al. 2004). In the tail, the temperature of the cold component is comparable to the hot component of the disk. The hot component corresponds to the intracluster medium. About $60 \%$ of the emission comes from the hotter spectral component.

\subsection{NGC 4501}

NGC 4501 shows a moderately extended hot gas halo (Fig. 5). The northeastern side is more extended than the southwestern side. X-ray emission is found up to $\sim 3^{\prime}(\sim 15 \mathrm{kpc})$ from the galactic disk north from the galaxy center. This X-ray plume is close to the $\mathrm{H} \alpha$-bright spiral arm at the outer northwestern disk, which has no counterpart in the southeast.

Due to the limited number of counts, the X-ray spectra of the disk and the tail (Fig. 6) has been fitted by a single hot component of $\sim 0.4 \mathrm{keV}$ and $\sim 0.7 \mathrm{keV}$, respectively (Table 3 ).

\section{Discussion}

To put the observed X-ray emission and spectra of the three galaxies into context, we first give a brief summary of the evolutionary stage of each galaxy.

\subsection{NGC 4569}

The spiral galaxy NGC 4569 is a very peculiar member of the Virgo cluster. Its very large size $\left(D_{25}=9.5^{\prime}\right.$, or $\left.47 \mathrm{kpc}\right)$ compared to other cluster members and negative radial velocity $\left(v_{\mathrm{r}}=-235 \mathrm{~km} \mathrm{~s}^{-1}\right.$ with respect to the cluster mean velocity of $\sim 1100 \mathrm{~km} \mathrm{~s}^{-1}$ ) have led to some doubt about its cluster membership, despite its close projected distance to the cluster center $\left(d=1.7^{\circ}=505 \mathrm{kpc}\right)$. Van den Bergh (1976) classified this very red galaxy as anemic due to its low arm-interarm contrast on optical images. The Hi line synthesis observations (Warmels 1988; Cayatte et al. 1990) showed that it has lost more than $90 \%$ of its HI gas and that its Hi distribution is heavily truncated. NGC 4569 is thus an exceptional galaxy, because it is one of the largest and most Hi deficient galaxies in the Virgo cluster (Solanes et al. 2001).

More recent $\mathrm{HI}$ observations have shown a low surfacedensity arm in the west of the galaxy, whose velocity field is distinct from that of the overall disk rotation. The observed gas distribution, velocity field, and velocity dispersion are consistent with a major stripping event that took place about $300 \mathrm{Myr}$ ago. 
M. Weżgowiec et al.: Hot gas in Mach cones around Virgo cluster spiral galaxies

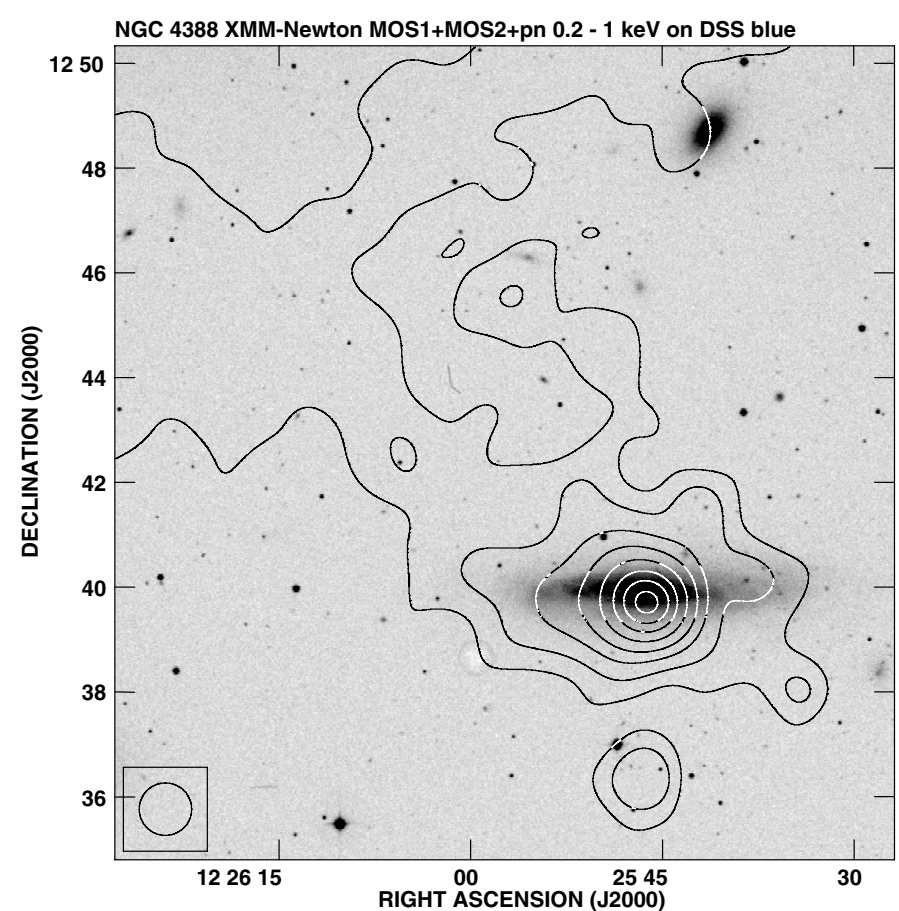

NGC $4388 \mathrm{H}$ I on XMM-Newton MOS1+MOS2+pn 0.2 - $1 \mathrm{keV}$

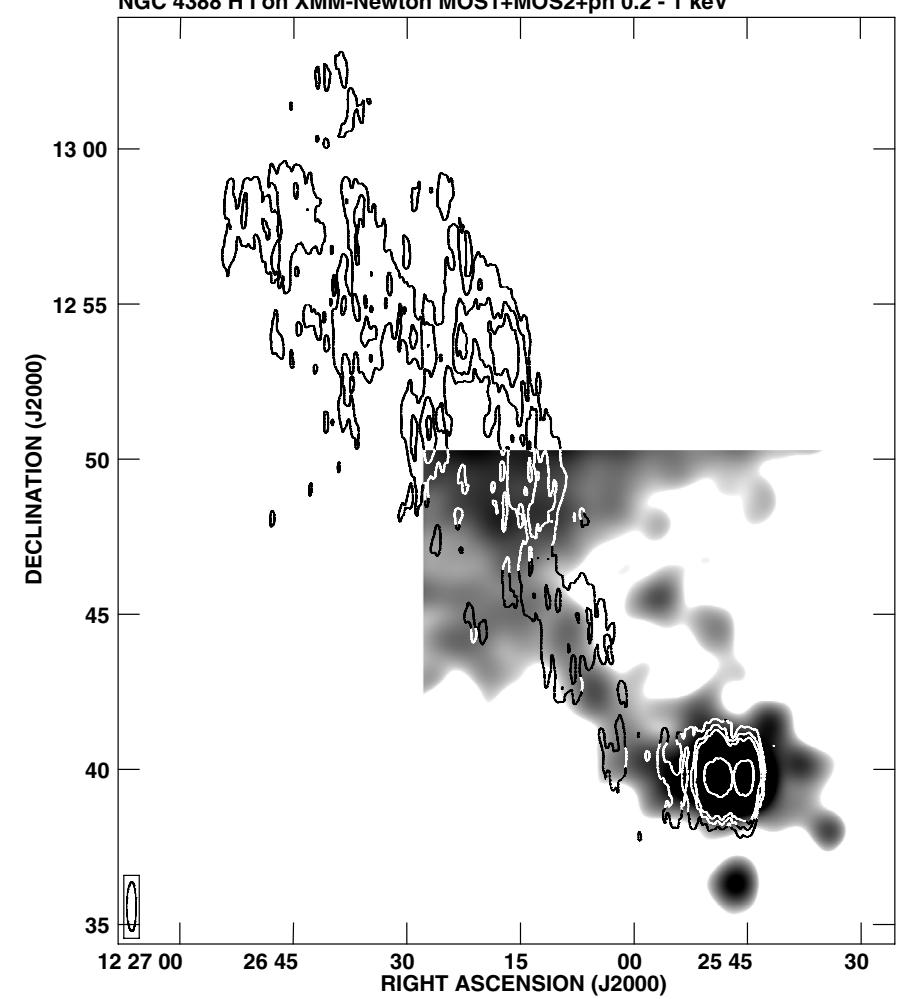

Fig. 3. X-ray emission (0.2-1 keV) of NGC 4388, together with an optical $B$ band image (upper panel) and the Hi map from Oosterloo \& van Gorkom (2005; lower panel). The X-ray map was cut at the position, where contribution from the M 86 halo starts to dominate. The first greyscale corresponds to 7 times the rms noise level.

This post-stripping scenario can reproduce the main observed characteristics of NGC 4569 (Vollmer et al. 2004). The analysis of the multiwavelength photometry of the galactic disk including GALEX UV observations (Boselli et al. 2006) confirmed this scenario. Tschöke et al. (2001) find a diffuse soft X-ray component extended to the west coinciding with a giant $\mathrm{H} \alpha$ structure.
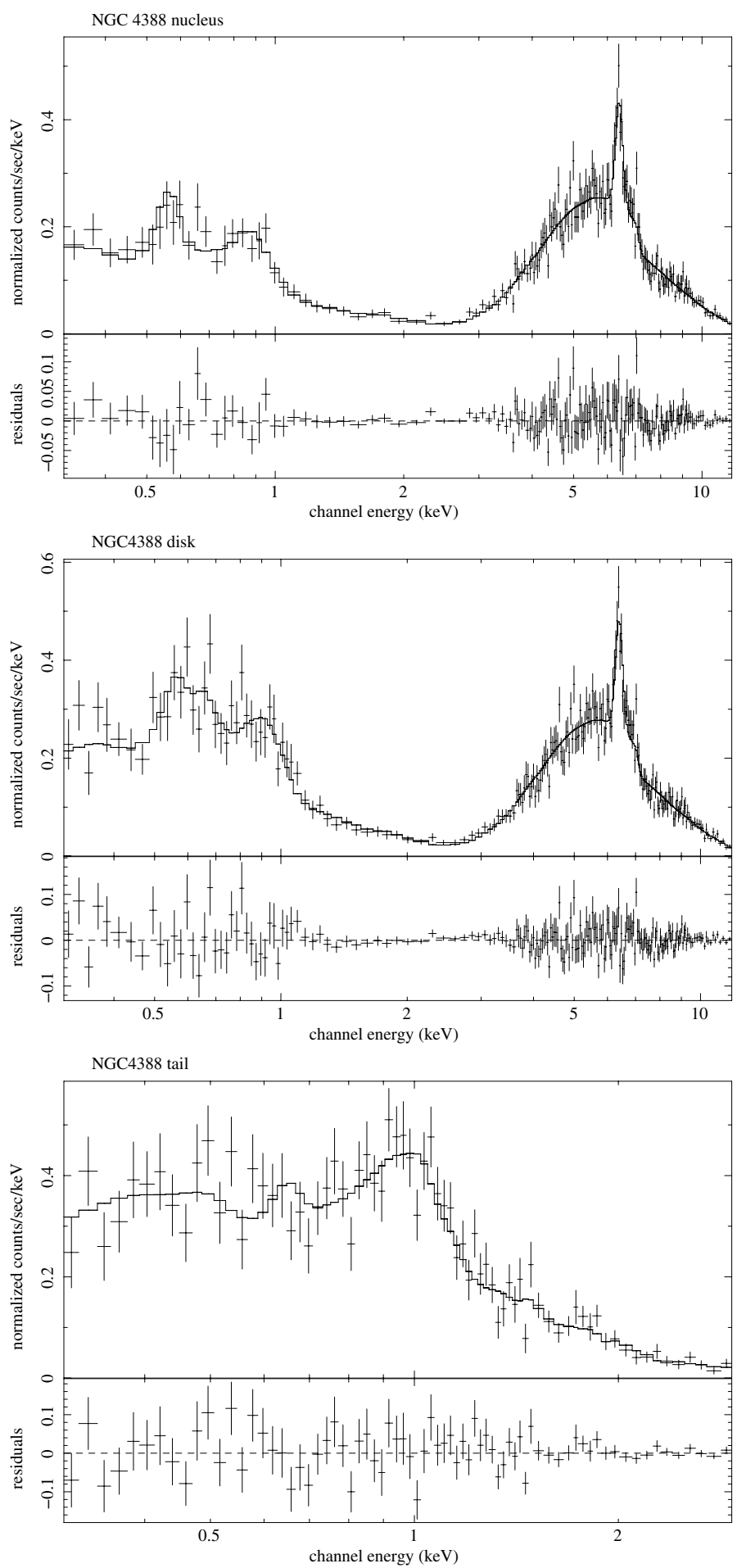

Fig. 4. The X-ray spectra of NGC 4388's nucleus (upper panel), disk and nucleus (middle panel), and tail (lower panel).

They conclude that the X-ray gas traces a large-scale outflow from accumulating supernova explosions and stellar winds in the galactic center.

Surprisingly, large radio continuum lobes have been found in NGC 4569 by Chyży et al. (2006). These lobes extend up to $24 \mathrm{kpc}$ from the galactic disk and are the first observed in a cluster spiral galaxy. Since the radio lobes are rather symmetric, they appear to resist ram-pressure. The most plausible explanation for the origin of the radio lobes is a galactic superwind. 

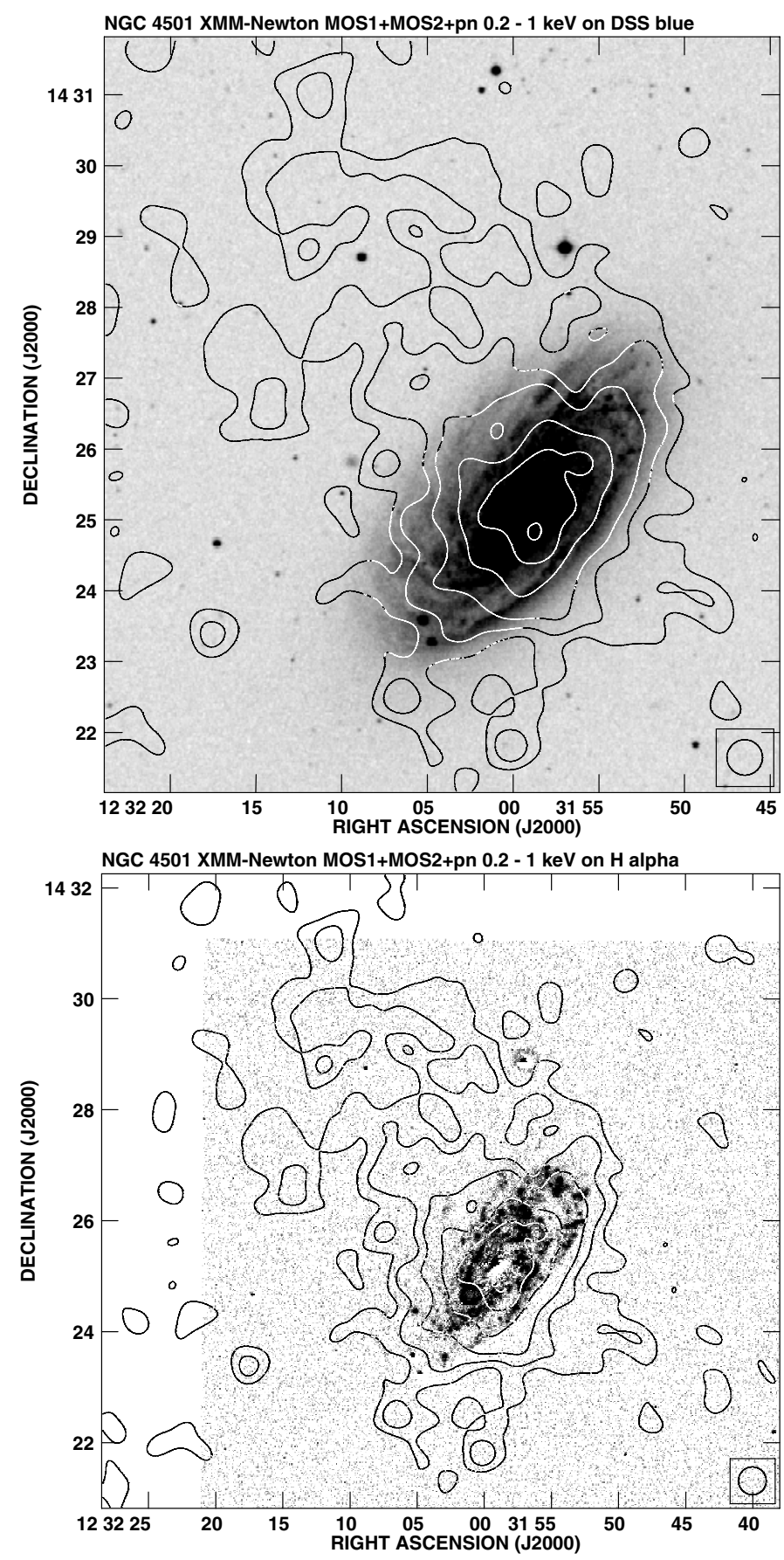

Fig. 5. X-ray emission (0.2-1 keV) of NGC 4501 overlaid onto an optical $B$ band image (upper panel) and the $\mathrm{H} \alpha$ image from Goldmine (lower panel). The map resolution is $30^{\prime \prime}$. The beam size is shown in the bottom right corner of the figure. The X-ray contours are 3, 5, 8, 16, 25 times the rms noise level.

To overcome ram-pressure, the pressure within the region of the radio lobes has to be higher than ram-pressure. Assuming the minimum energy condition, the total pressure of cosmic rays and magnetic fields in the lobes is $p \sim 10^{-12} \mathrm{dyn}^{-2}$. With a mean outflow velocity of $v_{\text {wind }}=700 \mathrm{~km} \mathrm{~s}^{-1}$, the mean density is $n \sim 3 \times 10^{-4} \mathrm{~cm}^{-3}$ (Chyży et al. 2006). A strong enough superwind-type flow can easily overcome the gravitational potential of the galaxy and resist ram-pressure. The fact that the radio lobes are entirely engulfed by the diffuse X-ray halo of NGC 4569 lends support to this scenario.
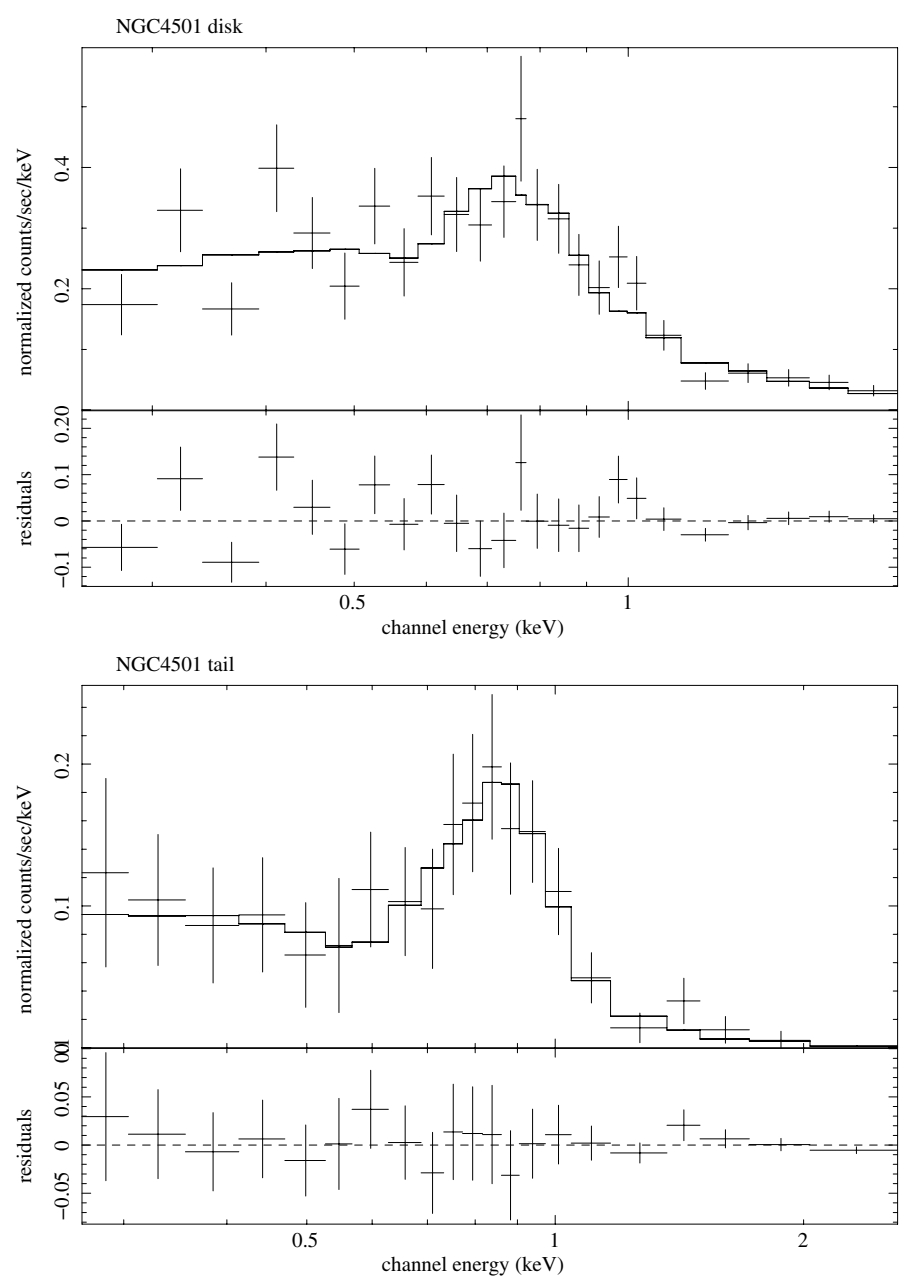

Fig. 6. The X-ray spectra of NGC 4501's disk (upper panel) and tail (lower panel).

The thermal pressure from the halo X-ray spectrum (Fig. 2) can be compared to the pressure of the cosmic rays and magnetic fields from Chyży et al. (2006). We find a pressure of $p_{\text {th }}^{\text {cold }} \sim$ $2 \times 10^{-13} \eta^{-0.5} \mathrm{dyn} \mathrm{cm}^{-2}$ for the cold component $\left(T=2 \times 10^{6} \mathrm{~K}\right)$

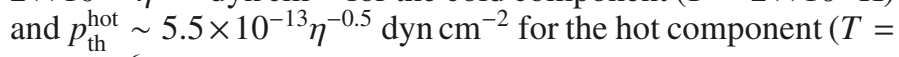
$5.7 \times 10^{6} \mathrm{~K}$ ), where $\eta$ is the volume filling factor. The thermal pressure of the hot phase is thus comparable, but a factor of 2 lower than that of the cosmic rays and magnetic fields. Thus the superwind might be driven by cosmic rays.

The triangular shape of NGC 4569's diffuse X-ray halo calls that of a bow shock. From the dynamical model of Vollmer et al. (2005) and Vollmer (2009), we know the direction of the galaxies motion within the intracluster medium. With an estimated galaxy velocity of $v_{\text {gal }}=1500 \mathrm{~km} \mathrm{~s}^{-1}$ and a sound speed of $c=550-700 \mathrm{~km} \mathrm{~s}^{-1}$ (depending on the adiabatic index) in the intracluster medium, the Mach number is $M \sim 2.1-2.7$ and the angle of the Mach cone (half of the opening angle) is $\alpha=\sin ^{-1}\left(\frac{1}{M}\right)=22-28^{\circ}$. On the other hand, since the very tenuous $\left(n \sim 10^{-4} \mathrm{~cm}^{-3}\right)$ intracluster medium is fully ionized and magnetized, the sound speed has to be replaced by the magnetosonic velocity $v_{\mathrm{ms}}=\sqrt{\left(c^{2}+v_{\mathrm{A}}^{2}\right)}$, where the Alfvén speed is $v_{\mathrm{a}}=\frac{B}{\sqrt{4 \pi n m_{\mathrm{p}}}}$. Typical magnetic field strengths in galaxy clusters are a few $\mu \mathrm{G}$ (Ferrari et al. 2008). 


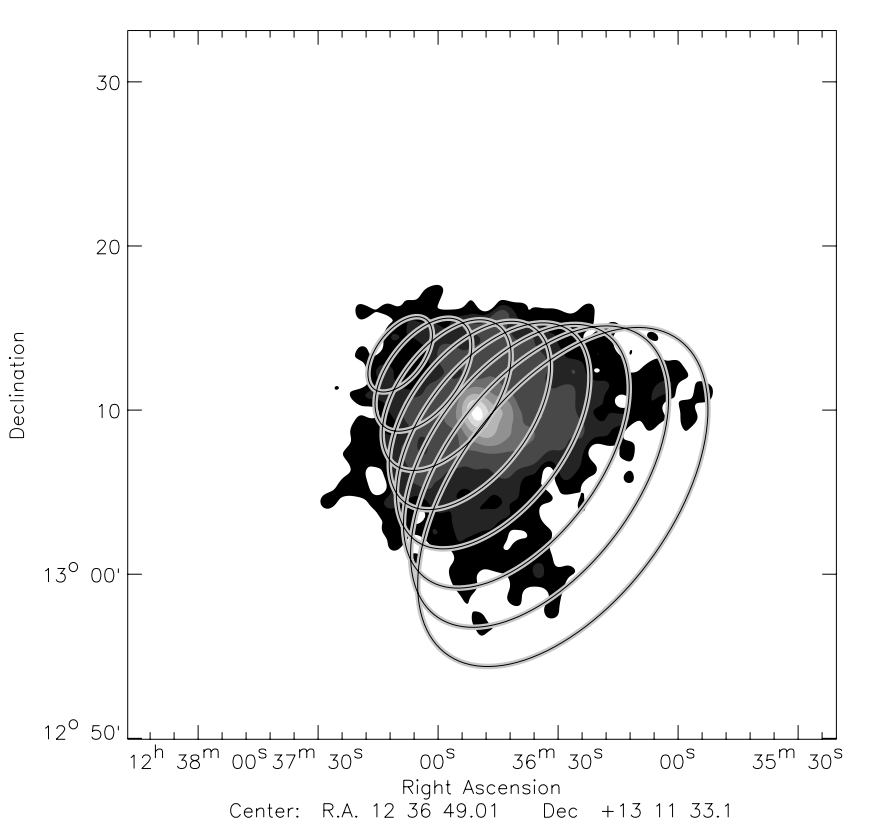

Fig. 7. X-ray emission $(0.2-1 \mathrm{keV})$ of NGC 4569 , together with the Mach cone whose direction is based on the dynamical model and whose opening angle is adjusted to the X-ray distribution.

We overlaid a Mach cone on the X-ray emission distribution of NGC 4569 in Fig. 7. The direction is based on the dynamical model, whereas the opening angle is fitted by eye to the triangular form of the X-ray distribution. In this way we find an apparent opening angle of $75^{\circ}$ or an apparent Mach cone angle of $\alpha=37.5^{\circ}$. The true Mach cone angle depends on the projection under which the Mach cone is observed, i.e. the angle $\beta$ between the galaxy's normalized 3D velocity vector and the plane of the sky.

Given the normalized 3D model velocity vector $(-0.64,0.53,0.56)$ (Vollmer 2009), this angle is $\beta=34^{\circ}$ and the true Mach cone angle is $30^{\circ}$. For $\beta=20^{\circ} / 40^{\circ}$ the true Mach cone angles are $36^{\circ}$ and $28^{\circ}$, respectively. NGC 4569's high radial velocity with respect to the Virgo cluster mean favors higher values of $\beta$. The true Mach cone angle is thus consistent with, but might be somewhat higher than, our estimates based on the sound speed of the intracluster medium. We thus suggest that the magnetic field plays a role and that the magnetosonic velocity is relevant to determining the Mach number. Based on true Mach cone angles between $30^{\circ}$ and $32^{\circ}$, a sound speed of $550-700 \mathrm{~km} \mathrm{~s}^{-1}$, a galaxy velocity of $1500 \mathrm{~km} \mathrm{~s}^{-1}$, and assuming an intracluster density of $n=10^{-4} \mathrm{~cm}^{-3}$, we derive Alfvén speeds between $300 \mathrm{~km} \mathrm{~s}^{-1}$ and $600 \mathrm{~km} \mathrm{~s}^{-1}$ and a magnetic field strength of $\sim 1-3 \mu \mathrm{G}$ at the location of NGC 4569 . The derived range of Alfvén speeds is thus comparable to that of the sound speed. The derived magnetic field strength is consistent with typical magnetic field strengths in galaxy clusters (Ferrari et al. 2008).

\subsection{NGC 4388}

NGC 4388's projected location within the Virgo cluster is close to $\mathrm{M} 87\left(1.3^{\circ}=385 \mathrm{kpc}\right)$. Yoshida et al. $(2002)$ discovered an $\mathrm{H} \alpha$ plume that extends up to $35 \mathrm{kpc}$ northeast of the galaxy center. Based on a Effelsberg $100 \mathrm{~m}$ telescope $\mathrm{HI}$ detection of

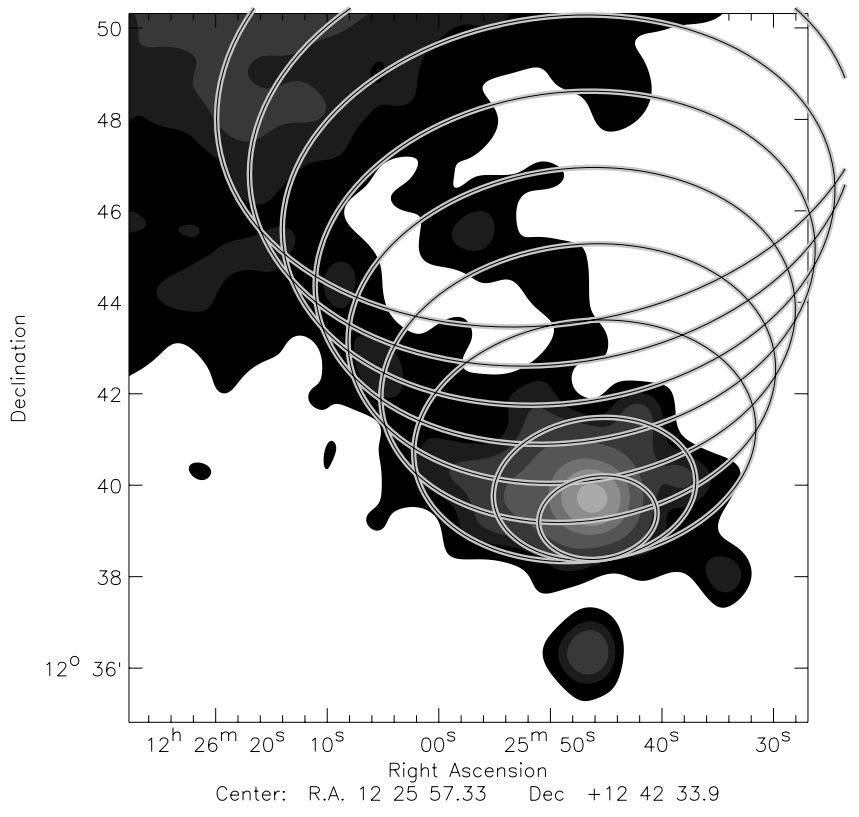

Fig. 8. X-ray emission $(0.2-1 \mathrm{keV})$ of NGC 4388 , together with the Mach cone whose direction is based on the dynamical model and whose opening angle is adjusted to the X-ray distribution.

the H $\alpha$ plume, Vollmer \& Huchtmeier (2003) made dynamical models and concluded that ram-pressure stripping can account for the observed extraplanar $\mathrm{H} \alpha$ and $\mathrm{HI}$ emission. Oosterloo \& van Gorkom (2005) observed NGC 4388 with the WSRT and discovered an $\mathrm{HI}$ tail that extends more than $100 \mathrm{kpc}$ to the north.

We clearly detect X-ray emission associated to the HI and $\mathrm{H} \alpha$ tail of NGC 4388. We can only attribute the observed X-ray emission to the first $\sim 5^{\prime}(\sim 25 \mathrm{kpc})$ of the $\mathrm{HI}$ tail which is about five times longer. This is because the projected $\mathrm{HI}$ distribution approaches M 86, and the halo of M 86 dominates the X-ray emission in this region.

As for NGC 4569 we adjusted a Mach cone to the X-ray and Hi emission distribution with the direction based on the dynamical model (Vollmer \& Huchtmeier 2003, Vollmer 2009). The apparent Mach cone angle is $\alpha \sim 26^{\circ}$ (Fig. 8). With a normalized 3 D model velocity vector $(0.09,-0.75,0.66)$ (Vollmer 2009$)$ the true Mach cone angle is $20^{\circ}$. This corresponds to a Mach number of 2.9 and a galaxy velocity of $\sim 1600-2000 \mathrm{~km} \mathrm{~s}^{-1}$ (with a sound speed of 550-700 $\mathrm{km} \mathrm{s}^{-1}$ ), which is consistent with the estimated galaxy velocity based on the dynamical model $\left(1700 \mathrm{~km} \mathrm{~s}^{-1}\right.$, Vollmer 2009). To obtain an Alfvénic Mach number of 2.9 with $v_{\text {gal }}=1700 \mathrm{~km} \mathrm{~s}^{-1}$, a magnetic field strength of $\sim 3 \mu \mathrm{G}$ is needed with an intracluster density of $n=10^{-4} \mathrm{~cm}^{-3}$.

Within the X-ray tail we measure a temperature of $0.87 \mathrm{keV}$ $\left(10^{7} \mathrm{~K}\right)$ and a density of $n \sim \eta^{-0.5} 10^{-3} \mathrm{~cm}^{-3}$. The thermal pressure is thus $p_{\text {th }}=1.4 \times 10^{-12} \mathrm{dyn} \mathrm{cm}^{-2}$, whereas ram-pressure is about 4-8 $\times 10^{-12} \mathrm{dyn} \mathrm{cm}^{-2}$. As in the halo of NGC 4569, the thermal pressure based on the X-ray observations is significantly lower than the estimated ram-pressure acting on NGC 4388.

We made an attempt to calculate the total mass of the hot $\mathrm{X}$-ray gas within the whole Hi tail. Unfortunately, this is not directly possible owing to the dominating X-ray halo of M 86. Several assumptions have to be made: first, a constant surfacebrightness along the whole tail is assumed. Next, the flux from the area of detectable X-ray tail is rescaled to the total volume 
of the Hi tail. Since the Hi tail is $\sim 3$ times longer than the X-ray tail, this leads to a three times larger volume.

Given the temperature from the spectral fit, the density of the hot gas is $n \sim \eta^{-0.5} 10^{-3} \mathrm{~cm}^{-3}$. A total gas mass of $\sim 2 \times$ $10^{8} M_{\odot}$ is found in the part of the X-ray tail that is not dominated by M 86's halo (south to the declination 124800 ). Since our volume estimate is on the high side and since we expect a volume filling factor below 1, a more realistic mass estimate of the X-ray tail is $\sim 10^{8} M_{\odot}$. Within a three times longer tail, there might be a few $10^{8} M_{\odot}$ of hot gas associated with the entire Hi tail of NGC 4388. This total mass of hot gas is comparable to the total mass of atomic hydrogen in the tail $\left(3.4 \times 10^{8} M_{\odot}\right.$; Oosterloo \& van Gorkom 2005). The total gas mass of the tail $\left(\sim 6 \times 10^{8} M_{\odot}\right)$ is much lower than the expected stripped gas mass based on the Hi deficiency $\left(2 \times 10^{9} M_{\odot}\right.$; Vollmer \& Huchtmeier 2007).

Possible reasons for this discrepancy are strong ISM-ICM mixing with a strong decrease in the gas density or star formation within the gas tail. Based on the reconstructed star formation history from deep optical spectra, Pappalardo et al. (2010) find that the stripped gas left the galactic disk $200 \mathrm{Myr}$ ago. The gas stripped from the location where the optical spectra were taken is presumably now in middle of the Hi tail. The gas within the tail thus traveled with a velocity in the sky plane of $\sim 300 \mathrm{~km} \mathrm{~s}^{-1}$. With a radial velocity of $\sim 300 \mathrm{~km} \mathrm{~s}^{-1}$, the total outflow velocity of the stripped gas is $\sim 400-450 \mathrm{~km} \mathrm{~s}^{-1}$. This 3D configuration is consistent with the Vollmer \& Huchtmeier (2003) model. The stripping timescale is reasonable in a scenario where NGC 4388 has been stripped by a recent passage through the Virgo cluster core, close to M 87 (Vollmer 2009). Therefore, we think that the tail traces the entire stripping process. Given the small number of HII regions within the Hi tail (Yoshida et al. 2004), gas loss via star formation does not seem to be significant.

Vollmer \& Huchtmeier (2007) suggested an alternative explanation for the missing stripped gas: spiral galaxies entering a cluster may rapidly lose the atomic gas that is located beyond the optical radius. This gas is (i) less bound than the gas in the inner disk and (ii) no longer stirred by strong interstellar turbulence, and it can easily be heated and evaporated. The stripping of the outer gas disk might happen before the galaxy enters the cluster core. In this case the galaxy already shows an initial HI deficiency of 0.4 before the stripping event. If such an initial HI deficiency is assumed, the sum of the observed Hi and extrapolated X-ray gas mass might account for all of the stripped gas. Alternatively, a fraction of the gas mass might be in the form of tenuous ionized gas with temperatures below $10^{6} \mathrm{~K}$.

\subsection{NGC 4501}

Polarized radio emission studies show a strong compression of the magnetic field at the southwestern edge of the galactic disk (Weżgowiec et al. 2007; Vollmer et al. 2007). NGC 4501's HI disk is sharply truncated to the southwest, well within the stellar disk. On the opposite side, there is low surface-density gas, which is more extended than the main $\mathrm{HI}$ disk. A detailed comparison of observations with a sample of dynamical simulations show that ram-pressure stripping can account for the main observed characteristics of NGC 4501 (Vollmer et al. 2008). The galaxy is stripped nearly edge-on, is heading southwest, and is 200-300 Myr before peak ram-pressure, i.e. its closest approach to $\mathrm{M} 87$.

A possible Mach cone is adjusted to the X-ray emission distribution in Fig. 9. The apparent Mach cone angle is $\sim 30^{\circ}$. With a normalized $3 \mathrm{D}$ model velocity vector $(0.48,-0.44,0.76)$ (Vollmer 2009), the true Mach cone angle is $20^{\circ}$ implying a

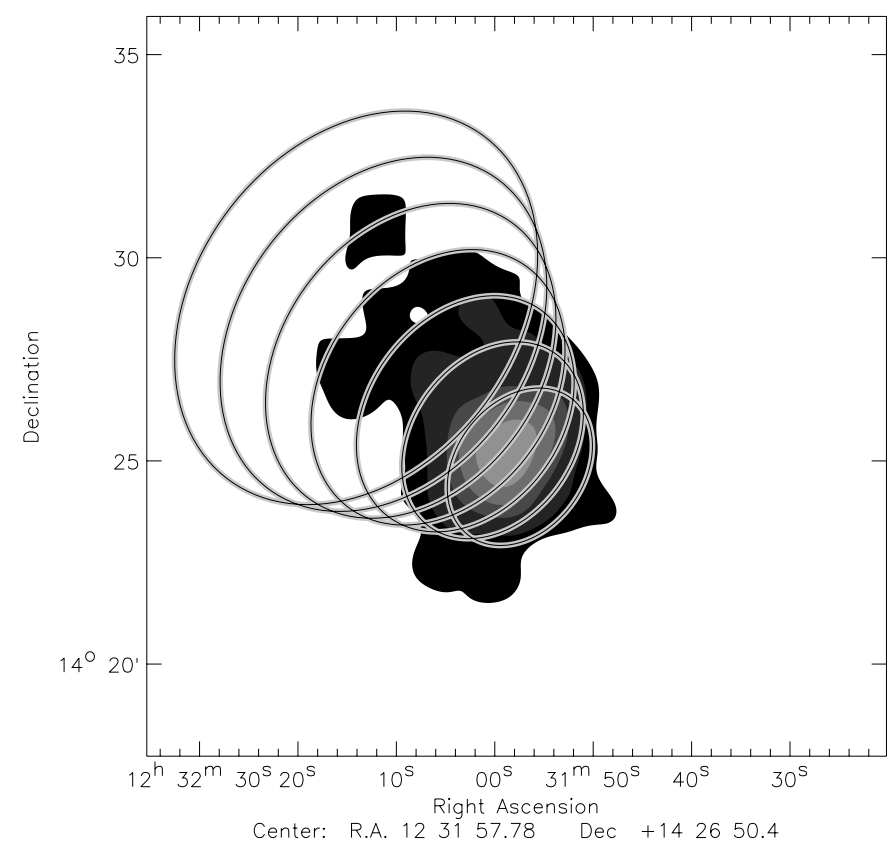

Fig. 9. X-ray emission (0.2-1 keV) of NGC 4501, together with the Mach cone whose direction is based on the dynamical model and whose opening angle is adjusted to the X-ray distribution.

Mach number of $M \sim 2.9$. Assuming a lower angle between the galaxy's normalized 3D velocity vector and the plane of the sky $\left(\beta=37^{\circ}\right.$ instead of $\left.49^{\circ}\right)$ yields a true Mach cone angle of $25^{\circ}$ and a Mach number of $M \sim 2.4$. If the sound speed is relevant for the Mach number, the galaxy velocity is $v_{\text {gal }} \sim 1300$ $2000 \mathrm{~km} \mathrm{~s}^{-1}$. This is consistent with the galaxy velocity based on the dynamical model $\left(v_{\text {gal }}=1500 \mathrm{~km} \mathrm{~s}^{-1}\right)$. If the Alfvén velocity exceeds the sound speed, a magnetic field strength of $\sim 2$ $3 \mu \mathrm{G}$ is needed when assuming an intracluster medium density of $n=10^{-4} \mathrm{~cm}^{-3}$.

Our X-ray data do not provide enough counts to obtain a high signal-to-noise spectrum. Therefore, we fitted a single temperature model to the X-ray spectrum of the tail and found the temperature to be $\sim 0.7 \mathrm{keV}$. This temperature is comparable to the temperatures of the hot extraplanar gas in NGC 4569 (0.49 keV) and NGC $4388(0.87 \mathrm{keV})$. The X-ray emission beyond the $\mathrm{HI}$ disk might come from stripped atomic gas mixed with the intracluster medium or a galactic wind due to star formation in the prominent northwestern spiral arm (Fig. 5).

\subsection{Hot gas within the Mach cones}

For NGC 4569 we have the lucky situation that the Mach cone is entirely filled by a galactic outflow. A galactic outflow encountering the ICM flow within the Mach cone should produce an observable contact discontinuity (see, e.g., Heinz et al. 2003; Kraft et al. 2011) in addition to the bow shock. According to Kraft et al. (2011) and Vikhlinin et al. (2001), the separation between the two features is $\sim 0.15$ times the distance between the galaxy center and the contact discontinuity for large Mach numbers. Assuming this distance to be $25 \mathrm{kpc}$, the projected distance between the bow shock and a contact discontinuity is about $45^{\prime \prime}$. This is close to the image resolution of $1^{\prime}$. That we do not observe two distinct features might be caused by the absence of a contact discontinuity, because the ICM flow within the Mach cone is turbulent, or might be due to resolution/sensitivity and/or projection effects. 
On the other hand, the Mach cone geometries of NGC 4388 and NGC 4501 are tentative. All extraplanar X-ray emitting hot gas is found within the Mach cones of the three galaxies.

Whereas it is most likely that the diffuse X-ray halo of NGC 4569 (Fig. 1) comes from a galactic superwind originating in a nuclear starburst, the extraplanar X-ray emission of NGC 4388 is most likely due to the mixing of stripped ISM with the hot intracluster medium. Typical temperatures of the cold and hot phase in the driven by star formation outflow of NGC 253 are $\sim 0.1 \mathrm{keV}$ and $\sim 0.3 \mathrm{keV}$, respectively (e.g. Bauer et al. 2008). The appearance of a "multi-temperature" halo is a natural consequence of the plasma driven out of collisional ionization equilibrium (CIE) towards a nonequilibrium ionization (NEI) state in a galactic outflow. The temperature of the hottest component in galactic outflows of massive spiral galaxies is about $0.4 \mathrm{keV}$ (Martin 1999).

The gas in NGC 4388's and NGC 4501's tails is significantly hotter than for typical galactic outflows of massive spiral galaxies. On the other hand, the X-ray tail of ESO 137-001 in ACO 3627 has a temperature of $0.7 \mathrm{keV}$ (Sun et al. 2006). The temperature of the X-ray tail of ESO 137-002 might be as high as $2 \mathrm{keV}$ (Sun et al. 2010). Both galaxies evolve in a galaxy cluster whose intracluster medium is three times hotter than that of the Virgo cluster (2.4 keV, Böringer et al. 1994). We suggest that the higher gas temperatures observed in the X-ray tails of NGC $4388(0.9 \mathrm{keV})$ and NGC $4501(0.7 \mathrm{keV})$ are due to the mixing of the stripped ISM into the hot intracluster medium of the Virgo cluster.

\section{Conclusions}

$X M M-N e w t o n$ observations of three Virgo cluster spirals have been presented. These galaxies are part of the ram-pressure stripping time sequence of Vollmer (2009), when NGC 4501 is still approaching the cluster center, and NGC 4388 and NGC 4569 have experienced peak ram-pressure $\sim 150 \mathrm{Myr}$ ago and 300 Myr ago. We find extraplanar diffuse X-ray emission in all galaxies.

- In NGC 4569 this emission is associated with the galactic outflow observed in $\mathrm{H} \alpha$ and radio continuum (Chyży et al. 2006). The diffuse $X$-ray emission has a triangular shape.

- In NGC 4388 the X-ray emission follows the beginning of the Hi tail discovered by Oosterloo \& van Gorkom (2003). The halo of M 86 dominates the X-ray emission at greater distances from the galaxy.

- In NGC 4501 it is located opposite the side of the galactic disk where the gas is compressed by ram-pressure (Vollmer et al. 2008).

Based on the 3D velocity vectors from dynamical modeling (Vollmer 2009), a simple Mach cone can be fitted to the triangular shape of NGC 4569's diffuse X-ray emission. To our knowledge this is the first direct evidence of a Mach cone in a cluster spiral galaxy. We can only observe it, because it is filled with hot gas from a galactic superwind. Assuming that all extraplanar diffuse X-ray emission has to be located inside the Mach cone, we also fit Mach cones to NGC 4388 and NGC 4501.

For NGC 4569 it is hard to reconcile the derived Mach cone opening angle with a Mach number based on the sound speed alone. Instead, a Mach number involving the Alfvénic speed seems to be more appropriate, yielding a magnetic field strength of $\sim 1-3 \mu \mathrm{G}$ for an intracluster medium density of $n \sim 10^{-4} \mathrm{~cm}^{-3}$. The shock is thus caused by fast magnetosonic waves.
Gas densities and temperatures are derived from the X-ray spectra. In the outflow of NGC 4569 the thermal pressure is a factor of 2 lower than the cosmic ray gas pressure. The outflow might thus be driven by cosmic rays. The thermal pressure in NGC 4388's X-ray tail is significantly less than the ram-pressure that NGC 4388 is experiencing. Whereas the temperature of the hot component of NGC 4569's X-ray halo $(0.5 \mathrm{keV})$ is at the high end but still typical of a galactic outflow, the temperature of the hot-gas tails of NGC 4388 and NGC 4501 are significantly hotter $(0.7-0.9 \mathrm{keV})$. We suggest that these higher gas temperatures result from the mixing of the stripped ISM into the hot intracluster medium of the Virgo cluster.

Acknowledgements. This work was supported by DLR Verbundforschung "Extraterrestrische Physik" at Ruhr-University Bochum through grant 50 OR 0801

\section{Appendix A: X-ray emitting volumes}

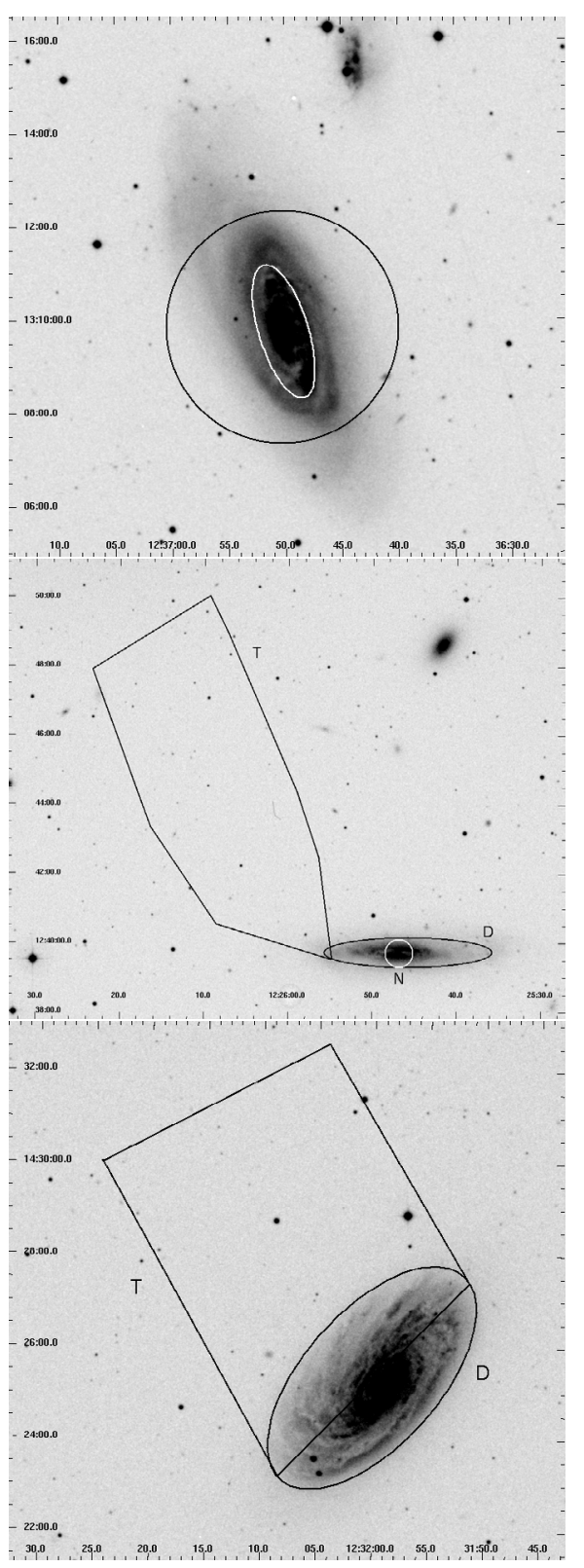

Fig. A.1. Regions for X-ray spectrum extraction and X-ray emitting volume calculations. 


\section{References}

Arnaud, K. A. 1996, Astronomical Data Analysis Software and Systems V, ed. G. Jacoby, \& J. Barnes, ASP Conf. Ser., 101, 17

Bauer, M., Pietsch, W., Trinchieri, G., et al. 2008, A\&A, 489, 1029

Böhringer, H., Briel, U. G., Schwarz, R. A., et al. 1994, Nature, 368, 828

Boselli, A., Boissier, S., Cortese, L., et al. 2006, ApJ, 651, 811

Carter, J. A., \& Read, A. M. 2007, A\&A, 464, 1155

Cayatte, V., van Gorkom, J. H., Balkowski, C., \& Kotanyi, C. 1990, AJ, 100, 604

Chung, A., van Gorkom, J. H., Kenney, J. D. P., \& Vollmer, B. 2007, A\&A, 659, 115

Chung, A., van Gorkom, J. H., Kenney, J. D. P., Crowl, H., \& Vollmer, B. 2009, AJ, 139, 2716

Chyży, K. T., Soida, M., Bomans, D. J., et al. 2006, A\&A, 447, 465

Ferrari, C., Govoni, F., Schindler, S., Bykov, A. M., \& Rephaeli, Y. 2008, SSRv, 134,93

Finoguenov, A., Briel, U. G., Henry, J. P., et al. 2004, A\&A, 419, 47

Gabriel, C., Denby, M., Fyfe, D. J., et al. 2004, ASPC, 314, 759

Heinz, S., Churazov, E., Forman, W., Jones, C., \& Briel, U. G. 2003, MNRAS, 346,13

Kaastra, J. S. 1992, An X-Ray Spectral Code for Optically Thin Plasmas, Internal SRON-Leiden Report, updated version 2.0

Kalberla, P. M. W., Burton, W. B., Hartmann, D., et al. 2005, A\&A, 440, 775

Kraft, R. P., Forman, W. R., Jones, C., et al. 2011, ApJ, 727, 41

Martin, C. L. 1999, ApJ, 513, 156

Mewe, R., Gronenschild, E. H. B. M., \& van den Oord, G. H. J. 1985, A\&AS, 62, 197

Oosterloo, T., \& van Gorkom, J. 2005, A\&A, 437, L19

Pappalardo, C., Lançon, A., Vollmer, B., et al. 2010, A\&A, 514, 33

Paturel, G., Petit, C., Prugniel, P., et al. 2003, A\&A, 412, 45

Rasmussen, J., Ponman T. J., \& Mulchaey J. S. 2006, MNRAS, 370, 453

Roediger, E., \& Brüggen, M. 2008, MNRAS, 388, 465
Roediger, E., Brüggen, M., \& Hoeft, M. 2006, MNRAS, 371, 609 Schulz, S., \& Struck, C. 2001, MNRAS, 328, 185

Soida, M., Otmianowska-Mazur, K., Chyży, K., \& Vollmer, B. 2006, A\&A, 458, 727

Solanes, J. M., Manrique A., Garcia-Gomez, C., et al. 2001, ApJ, 548, 97 Stevens, I. R., Acreman, D. M., \& Ponman, T. J. 1999, MNRAS, 310, 663 Strüder, L., Briel, U., Dennerl., K., et al. 2001, A\&A, 365, 18 Sun, M., \& Vikhlinin, A. 2005, ApJ, 621, 718

Sun, M., Jones, C., Forman, W., et al. 2006, ApJ, 637, L81

Sun, M., Donahue, M., Rödiger, E., et al. 2010, ApJ, 708, 946 Tschöke, D., Bomans, D. J., Hensler, G., \& Junkes, N. 2001, A\&A, 380, 40

Tonnesen, S., \& Bryan G. L. 2009, ApJ, 694, 789

Turner, M. J. L., Abbey, A., Arnaud, M., et al. 2001, A\&A, 365, 27 van den Bergh, S. 1976, ApJ, 206, 883

Veilleux, S., Bland-Hawthorn, J., Cecil, G., Tully, R. B. M., \& Scott T. 1999, ApJ, 520, 111

Vikhlinin, A., Markevitch, M., \& Murray, S. S. 2001, ApJ, 551, 160

Vollmer, B. 2009, A\&A, 502, 427

Vollmer, B., \& Huchtmeier, W. 2003, A\&A, 406, 427

Vollmer, B., \& Huchtmeier, W. 2007, A\&A, 462, 93

Vollmer, B., Cayatte, V., Balkowski, C., \& Duschl, W., J. 2001, ApJ, 561, 708

Vollmer, B., Balkowski, C., Cayatte, V., et al. 2004, A\&A, 419, 35

Vollmer, B., Soida, M., Otmianowska-Mazur, K., et al. 2006, A\&A, 453, 883

Vollmer, B., Soida, M., Beck, R., et al. 2007, A\&A, 464, L37

Vollmer, B., Soida, M., Chung, A., et al. 2008, A\&A, 483, 89

Wang, Q. D., Owen, F., \& Ledlow, M. 2004, ApJ, 611, 821

Warmels, R. H. 1988, A\&AS, 72, 57

Weżgowiec, M., Urbanik, M., Vollmer, B., et al. 2007, A\&A, 471, 93

Yoshida, M., Yagi, M., Okamura, S., et al. 2002, ApJ, 567, 118

Yoshida, M., Ohyama, Y., Iye, M., et al. 2004, AJ, 127, 90 